\title{
A Cloud-Based Experimental Teaching System for Network Engineering Specialty
}

\author{
Tang Junyong \\ School of computer science and engineering \\ Xi’an Technological University \\ Xi'an, China \\ tjy112@hotmail.com
}

\author{
Xiao Feng \\ School of computer science and engineering \\ Xi'an Technological University \\ Xi'an, China \\ xffriends@163.com
}

\begin{abstract}
In order to cultivate innovative and entrepreneurial talents for network engineering specialty, a novel experimental teaching system was designed. The fresh system adopted new methods promoting the students' study interest and enthusiasm. Three experimental steps were designed and each step provided special training function. By the mutual impact on three steps, an across-the-board and creative teaching mode was constructed step by step. Meanwhile, the system combines with the virtual technology of the cloud platform effectively to foster the students' ability of innovation and entrepreneur. The practice results demonstrate the teaching system to outperform the conventional experimental te aching modes.
\end{abstract}

Keywords-experimental teaching; ability of innovation and entrepreneur; cloud platform; virtual technology

\section{INTRODUCTION}

At the age of the Internet plus, the cloud technology and data mining are all rapidly applied to various fields, e.g. study, living and manufacturing aspects, as in [1]. How to cultivate personnel with the ability of innovation consciousness and grasping practical skills is the major objective of many universities. The network specialty of computer characterizes the combination of practice and theory, which leads the experiment teaching to the only way for fostering the person with high level of professional ability. The innovation of experimental method and the constructing of the experimental environment is the key factor to talent cultivation, as in [2-3]. Based on the research in the growing process of mental and various teaching methods, such as task driving, case teaching, guide teaching, proposed the novel design to the experiment teaching system based on the cloud platform. Aided by the cloud technology, the experiment mode is well received by the teachers and students. The teaching quality and effectiveness significantly improve.

\section{THE DESIGN IDEA OF THE EXPERIMENTAL SYSTEM}

The core idea of the experimental teaching system is that teachers present the experiment demands and documents with guide steps to students. Each student selects the specific test task according to respective ability. With regard to the task excess of the students' ability, teachers instruct on practical and

This research was supported partly by the Education Reform Foundation of Xi'an Technological University (No. 17JGZ10), the Reform of Graduate Education and Teaching Foundation (No. XAGDYJ170218), the Research Project on Teaching Reform of Education in Shaanxi Province (No. 17JY015) and the Characteristic Disciplines in Education Department of Shaanxi Province (No. 080901). appropriate teaching principal for students maximally to understand the trial information.

In the process of experiments implementing, the methods of a group study and the role-playing are introduced, by which the teacher checks students' study status with the assistance of a group leader. After the experiment lesson, the members of a group supervise each other whether one finishes the experimental task. It's very important for a teacher to construct a slack environment to give students relatively rich time. Students in the relaxed atmosphere would arrange study schedules and accomplish the experimental task reasonably utilizing spare time. The advantage of the design procedure is the interest and enthusiasm in the study to be aroused because the student promotes the active study ability by the means of supervising not only others but also himself.

For promoting the student's subjective initiative, the design of experiment schedules divides the experimental teaching into several parts: basic exercise, complex practical training and real case research and development, up to the most optimal teaching effect according to every student's specific study growth and recognition process. With the development of comprehension and recognition for the experimental project, the initiative to participate in the experiment also increases, as in [4]. Because the attention and participation of students are more and more, they find some shortcuts in the experimental system as well, and then propose some creative designs and concrete measurements to improve the effect of experiment teaching, which promotes the cultivation of the innovative ability to students.

Through the mutual action which includes the participation in the experimental system between students and teachers, the user of the system is not only a constructor but also a maintainer. Whoever innovatively constructs new experiment task excess of the requirements of teaching research is considered as the supplement. The function of the experimental system, therefore, accommodates more research projects, and the application scope covered by the system is also dynamically extended. The limit to the traditional relationship between teachers and students has become blurry. So a fresh relationship of objective and subjective is forming and constitutes a new type of experiment teaching. The construct diagram for them is shown in Fig. 1. 
In the second step, complicated practical training, the students flexibly finish the comprehensive experiments by applying the learned knowledge. The step also has an advantage which recombines with test instruments and resources to design and develop the intricate projects of network engineering. According to the students' preference, the experimental groups are divided to enhance the collaborative spirit and communication skills in order for students cooperatively researching the experiments. The self-evaluation in an inner group and mutual evaluation between groups are implemented with the suggestion of guide teachers after every experiment is finished. The important objective of the second step is that the students are fostered the ability in practice, communication, global thinking, integrative controlling and effectively resolving complex problems in network engineering.

The students in the third step, real case research and development could expand the experimental system more of fresh function available to the development of realistic networking engineering projects. Based on the comprehension of the experimental system, students propose the new requirements for implementing high-level experiments. Thereupon, the system becomes the powerful tool for students proficiently to develop available projects. When the students qualify the realistic project of network engineering, the teacher distributes the information about real engineering requirements. The students act according to the procedure as same as the normal experiment training, which includes all training tasks, for example, dividing groups, designating roles, cooperating research and development. Similarly, the evaluation of the real networking projects from in-group and groups immediately carried out. Thus, the experimental system is a position that cultivates the ability of innovation and creativity, and presents the talent of students. Everyone in a group is conscious of the deficiency and is clear about the improvements compared with other research groups.

In terms of the above analysis, the experimental system is different to the conventional experimental platform. It doesn't characterize the traits of customary experiments, e.g. demonstrating and validating, as gets rid of the limitation of time and space. So teaching methods could be implemented according to the students' traits and environments. The ability of the students, for example, activeness, initiative, and innovativeness, is promoted. The three steps blend the realistic experimental study and virtual network engineering environment for the convergence of theory and practice. The three steps are all linked with another, by which the experiment teaching creates an across-the-board and creative teaching mode step by step. At last, the system promotes the students' comprehensive quality, practice skills, and innovative ability in the network engineering specialty.

\section{The ARChItECtURE OF THE EXPERIMENT System BASED ON CLOUD}

\section{A. The Cloud Platform of the System}

Firstly, all equipment included software, virtual experiment instruments and documents in all three steps is stored on the cloud server, which realizes the sharing of information all time for fertilizing the resource spool. Secondly, the request for 
assigning the experiment resources and equipment is committed to the cloud according to the various experiment steps. Thus the processor in the cloud calls the strategy of allocating resources, and the scheduled resources are designated for users. Lastly, various kinds of experiments are implemented in the cloud client-terminal. Additionally, the cloud platform also has the function of online communication between teachers and students. It's convenient for users to discuss problems and clear up confusion. The procedures constructing a cloud platform are shown below:

- Constructing an experiment teaching system based on the cloud platform. The experimental environment is fertilized by requesting and allocating resources. The effect of experimental teaching is optimal with the assistance of online communication and dynamically virtual technology.

- Constructing an open resource pool with standardization and normalization. This procedure recombines flexibly the hardware and software for convenience normalizing the experimental operation. With the standard of operation and access to experiments, the experimental resources are shared out.

- Developing an excellently agile strategy of scheduling resources. The strategy not only meets the requirement of users for real-time using but also promotes the utilization of experimental resources.

- An efficient storage management mechanism is studied to realize real-time tracking of experimental process and record keeping of experimental data.

\section{B. Overall Architecture}

The experimental teaching system, which is based on cloud architecture, highlights the idea of study in practice to improve ability. The use of cloud platform technology realizes the function of mass storage, dynamic allocation of resources, the flexible application, collaborative work, autonomous learning, and the high-quality service, etc.

The experimental teaching system uses the open-source Hadoop distributed system infrastructure developed by the Apache Foundation to complete data processing operations in a highly reliable, efficient, and scalable way, as in [5]. The core of the Hadoop framework includes HDFS and Map Reduce. The function of HDFS is to store large amounts of data efficiently, and the function of the other is to perform data calculation and correlative mining operations. The Hadoop framework provides users with a more versatile cloud computing platform, and its open-source performance allows users easily to carry out secondary development. Efficient use of the distributed cloud platform, very conveniently, achieves massive data storage, mining and other operations.

The experimental teaching system based cloud platform is divided and constructed according to the three cloud technology of SaaS, PaaS, and IaaS, as in [6]. The SaaS corresponds to the cloud access layer, providing multi-desktop operation and experimental teaching services for teachers and students across platforms and integrated network projects. Users access multiple types of virtualized experimental environments by terminals such as computers, smart phones, and cloud devices. The SaaS involves 3 stages of experimental operation.

The PaaS technology corresponds to introduce the experimental system to cloud and has functions such as identity authentication, engine services, control of empirical resources and access, implementation of data mining, and construction of resource pools to provide technical support for the SaaS technology. Meanwhile, this technology includes experimental teaching management, correlative data mining, experimental resources and code servers, business engine of project development management, virtualized environment servers.

The IaaS technology corresponds to the hardware infrastructure layer and manages the physical hardware that provides a hardware platform to the experimental system in a cloud. It virtualizes the resource pool hardware and is the basis of smooth performance of the experimental system. The physical hardware includes server clusters, fiber switches, high-availability storage devices, and other servers. The technology of storage virtualization, network virtualization and other cloud virtualization in IaaS, achieves the management of users, security, computing, storage, monitoring, project resources and virtualization operations.

In summary, the experimental software resources are integrated into normal information and inputted to cloud servers to provide users with experimental services. The cloud platform also introduces a project development management and business service engine. Both the development application program and the development process, such as experimental teaching client and various projects, can directly access the platform through the SaaS to create a real working environment for users. At the same time, the platform uses the experience of resource virtualization method of cloud and deploys the hardware equipment required for experiments, as in [7]. On the cloud platform, a hardware resource pool is formed. Devices in the resource pool are stored in a physical manner and serve the user's application through the network environment. The hardware resource pool has good development, dynamic, and scalability, etc. These features maximize the user's request.

\section{Resource Scheduling and Configuration}

The below is the core idea of the system's resource scheduling and configuration.

- According to the specific needs of the experimental project and the actual situation of the user, the user submits a request for various types of resources, and uploads the request to the server responsible for the resource scheduling and configuration.

- After the server receives the resource request, it calls the dynamic resource allocation algorithm to implement resource allocation and scheduling.

- Resource allocation control system for users to configure resources and conduct supervision and management.

- Transfer the required resources from the resource pool to the user. 
At this point, the user has successfully obtained the resources he applied for.

\section{The Mining of Correlative Data}

The experimental teaching platform utilizes Map Reduce, a core technology provided by Hadoop distributed framework, to perform extraction and mining of correlative data on a large amount of collecting data set. In this way, teachers can master the study status of students and eliminate the drawback of traditional experiment about the dependence on teachers individually to upload and design experiments.

The advantage of mining correlative data fully arises in enthusiasm and initiative of students, by the novel experimental mode continuously enriching speculative data, and developing more effective and specific experimental programs in various stages. Using the mining technology innovates the experimental system by analyzing large amounts of stored data and digging out the correlation between the data onto teaching.

\section{The Practice of the Experimental Teaching} SYSTEM

Taking the core course of network engineering specialty, "Local Area Network design and application," as an example, compared with the conventional teaching mode, the experimental teaching based on the cloud platform shows an outperformance. The course just has 9 simple basic experiments. Almost about $60 \%$ of students can complete $5 \sim 6$ experiments; about $30 \%$ of students can complete $7 \sim 8$ experiments and only about $10 \%$ of students can finish all nine experiments. However, in the new experimental teaching system, nearly all the students can complete nine tests, and more than $95 \%$ of students can participate in the training of comprehensive complex projects. In addition, about $30 \%$ of students are qualified for the innovative research and development of real projects. Judging from the quality of the completed experiment, the student's basic experiment was fully passed, with an excellent rate of $38 \%$, a good rate of $55 \%$, a medium rate of $5.9 \%$, and a passing rate of $1.1 \%$. This shows that the student has mastered the basics of the course well.

Another example of the "Routing and Switching" course illustrated the teaching effectiveness. The teacher gives a welldesigned network topology in the traditional teaching mode, and 5 to 6 simpler configuration items are required to accomplish. The students can only meet the requirements of the experimental instruction book. However, for the powerful function of routers and switches, it is obviously impossible for 5-6 simple operations to include all their functions. At the same time, under the novel teaching system, the students can design their own network topology, autonomously design problems to be solved, plan and design the test goals and contents by themselves, and execute the experimental process.

Several students have used the system to apply for a virtual CCNP integrated development environment, then drawn the corresponding network topology, implemented the equipment configuration, and designed up to 20 tests of network troubleshooting and optimization based on the powerful functions of CISCO equipment and practical project requirements. These enriched experiments have transcended the requirements of the test syllabus. After the students extended and participated in the experimental system at cloud terminals, they meet more closely to the experimental teaching requirements. Their study statuses are more objectively reflected. Fig. 2 shows a comparison to the number of accomplishing extended experiments under the traditional model and the novel teaching system in a cloud.

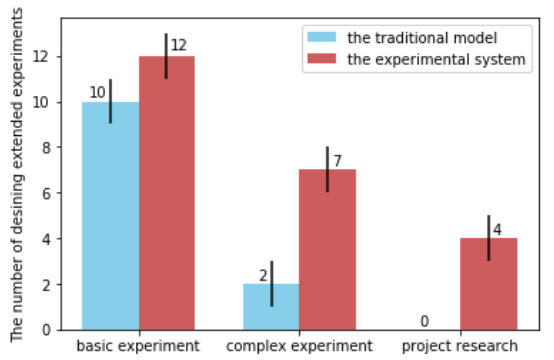

Fig. 2. The comparison of accomplishing extended experiments

\section{SUMMARY}

In order to cultivate innovative and entrepreneurial talents in network engineering specialty, with the help of today's advanced cloud technologies and fresh teaching methods, experimental teaching can play a greater role in cultivating students' practical skills and innovation capabilities. The cloud-based experimental teaching system also needs to extend and expand the contents from both the depth and the breadth, meets the new need of experimental teaching, and continues to innovate and improve from the aspects of platform resources, such as multiple styles, comprehensiveness, innovativeness and reform spirit.

\section{REFERENCES}

[1] YANG, JIANCHENG, et al. "Reform and Practice of Experimental Teaching of Mechanical Engineering Specialty in Education of Outstanding Engineers," 4th International Conference on Vehicle, Mechanical and Electrical Engineering (ICVMEE), Beijing, pp. 56-62, April 2017.

[2] Peng Ying Du,Hao Jiang,Guo Hai Ren,Xuan Chang Zhou. "Experimental Teaching Model on Information and Electrical Engineering Speciality,” Advanced Materials Research, vol. 271, pp. 1290-1293, March 2016.

[3] Tong Qing Wu,Nian Chun Xu,Lin Qing Huang. "Research Experimental Teaching System Based on Innovative Practice Ability,” Advanced Materials Research, vol. 671, pp. 2331-2335, Jue 2016.

[4] Jing XIAO. “Exploration of Experimental Teaching Reform of Public Finance,” Higher Education of Social Science, vol. 7, pp. 58-61, July 2016.

[5] Wu, T., Xu, N., \& Huang, L. "Research experimental teaching system based on innovative practice ability,” Advanced Materials Research, vol. 273, pp. 1580-1583, Sep 2016.

[6] Fry, D. J. “Teaching experimental design,” ILA R Journal, vol. 55, pp. 467-471, July 2016.

[7] Ibrahim H. Garbie. "Incorporating Sustainability/Sustainable Development Concepts in Teaching Industrial Systems Design Courses,” Procedia Manufacturing, vol 15, pp. 456-601, Aug 2017. 